\title{
Finding new physics, phenomenological, experimental, and astrophysical predictions from neutrino mass
}

\author{
C.R. Das ${ }^{1}$, Katri Huitu ${ }^{2}$, Zhanibek Kurmanaliyev ${ }^{3}$, \\ Bakytbek Mauyey $^{4}$ and Timo Kärkkäinen ${ }^{5}$ \\ ${ }^{1}$ Bogoliubov Laboratory of Theoretical Physics, Joint Institute for Nuclear Research, Dubna, \\ Moscow Region, Russian Federation \\ ${ }^{2}$ Department of Physics, University of Helsinki, Helsinki, Finland \\ ${ }^{3,4}$ Flerov Laboratory of Nuclear Reactions, Joint Institute for Nuclear Research, Dubna, \\ Moscow Region, Russian Federation \\ ${ }^{5}$ Institute for Theoretical Physics, ELTE Eötvös Loránd University, Budapest, Hungary \\ E-mail: ${ }^{1}$ das@theor.jinr.ru, ${ }^{2}$ katri.huitu@helsinki.fi, ${ }^{3}$ kurmanaliyev@jinr.ru, \\ ${ }^{4}$ bahytbek01@yandex.ru, ${ }^{5}$ karkkainen@caesar.elte.hu
}

\begin{abstract}
The crucial phenomenological and experimental predictions for new physics are outlined, where the number of problems of the Standard Model (neutrino masses and oscillations, dark matter, baryon asymmetry of the Universe, leptonic CP-violation) could find their solutions.

The analogies between the cosmological neutrino mass scale from the early universe data and laboratory probes are discussed and the search for new physics and phenomena.
\end{abstract}

\section{Introduction}

Despite being the most successful theory of particle physics to date, the Standard Model of particle physics is not perfect. According to the Standard Model, neutrinos are massless particles. However, neutrino oscillation experiments have shown that neutrinos do have mass. We do not know how to incorporate neutrinos into the standard model because we do not know if they are Dirac or Majorana fermions. But if you go beyond the Standard Model and allow neutrinos to have mass, which you have to do to be consistent with what we observed. It's not only allowed, but it's also arguable that it might be the best explanation possible.

Neutrino oscillation experiments measure atmospheric and solar neutrino-mass splittings and are getting very close to a determination of the neutrino mass ordering also $\mathrm{CP}$ violation among neutrinos including $\theta_{23}$ octant. However, neutrino oscillation experiments have no information about the absolute scale of the sum of total neutrino masses, $\Sigma m_{\nu}$.

Observational cosmology uses two methods to study the evolution and structure of the universe:

- the ancient photons that make up the $2.7 \mathrm{~K}$ cosmic microwave background (CMB) allow us to explore cosmological history as far back as a redshift of $\mathrm{z}=1400$, some 300,000 years after the Big Bang; 
- radiation from neutral hydrogen gas at a wavelength of 21-cm traces the large-scale distribution of matter and dark matter, which in turn probes dark energy, neutrino mass, etc.

Cosmological observables are not the only probes of the absolute neutrino mass scale, laboratory searches such as kinematic measurements in $\beta$-decay experiments and neutrino-less double- $\beta$ decay $(0 \nu 2 \beta)$ search also provide complementary information.

Detection of the absolute neutrino mass scale with cosmology would be crucial to test the consistency between different probes, an inconsistency would be an interesting indication of new physics [1].

\section{The KATRIN experiment}

Now, neutrino physics has entered an era of precision measurements. The KATRIN experiment is famous for the direct measurement of the mass of the neutrino before 2025 if its mass is greater than $0.20 \mathrm{eV}$ (at $5 \sigma$ only if it is greater than $0.35 \mathrm{eV}$ ). Their first result has just been published (after just four weeks of data collection): the neutrino mass is less than $1.1 \mathrm{eV}$ at $90 \% \mathrm{CL}$, which improves by a factor of two the previous direct estimate $(2.3 \mathrm{eV}$ from the Troitsk experiment in 2003). Of course, this value cannot compete with indirect cosmological measurements; With the data from Planck 2018, a value less than $0.12 \mathrm{eV}$ is estimated (actually the sum of the masses of the three neutrinos is limited). So, the most relevant thing about the recent result is that KATRIN seems to be working perfectly. This is not a small thing for such a complex and delicate experiment.

The most striking thing about the new result is the estimate of the square of the neutrino mass $m_{\nu}^{2}=-1.0_{-1.1}^{+0.9} \mathrm{eV}^{2}$ at $90 \%$ CL (you read correctly, it seems laughable, but it is a negative number). In fact, KATRIN can only directly measure the square of the neutrino's mass, from which the estimation of the mass of the most massive state of the neutrino is made. Of course, everyone knows that this negative value is an accident of the Monte Carlo method used for data analysis (which does not bias the analysis by assuming that the value must be positive). When the uncertainty of the measurements is reduced the central value will approach zero and finally, it will end up being positive, see Figure 1.

The measure of negative values of $m_{\nu}^{2}$ has made rivers of ink flow many times; perhaps you remember the tachyon nature of the neutrino after the LLNL experiments in 1995 or OPERA in 2011. But non-specialists should not be confused if the neutrino were tachyon, we would already know. The beauty of any experimental dossier lies in its systematics $[2,3,4]$.

\section{The GERDA experiment}

There are several ongoing experiments that try to elucidate this crucial question thanks to the observation of double beta decay without neutrinos $(0 \nu 2 \beta)$. The GERDA experiment publishes its latest result in Science, a half-life for the $T_{1 / 2}(0 \nu 2 \beta)$ process $>0.9 \times 10^{26}$ years $(90 \% \mathrm{CL})$; combined with other experiments implies, if the neutrino is Majorana, an upper limit to the mass of the neutrino of $0.07-0.16 \mathrm{eV}$. GERDA has obtained this result after not observing any signal in its germanium-76 detector after an exposure time of $82.4 \mathrm{~kg} \cdot$ year [5]. No signal has been observed, which allows obtaining a limit for the half-life of the decay $(0 \nu 2 \beta)$. The most important thing about GERDA is that its noise background is very clean, which ensures that its limit (even if it only reaches $2.4 \sigma$ ) is quite reliable, see Figure 2.

By the way, the Spanish NEXT-100 experiment has an estimated sensitivity (after five years of data collection) of $T_{1 / 2} \sim 1.1 \times 10^{26}$ years; then you can get a slightly better result than GERDA, the best so far [6]. In fact, $(0 \nu 2 \beta)$ events could only happen if neutrinos were Majorana particles. 


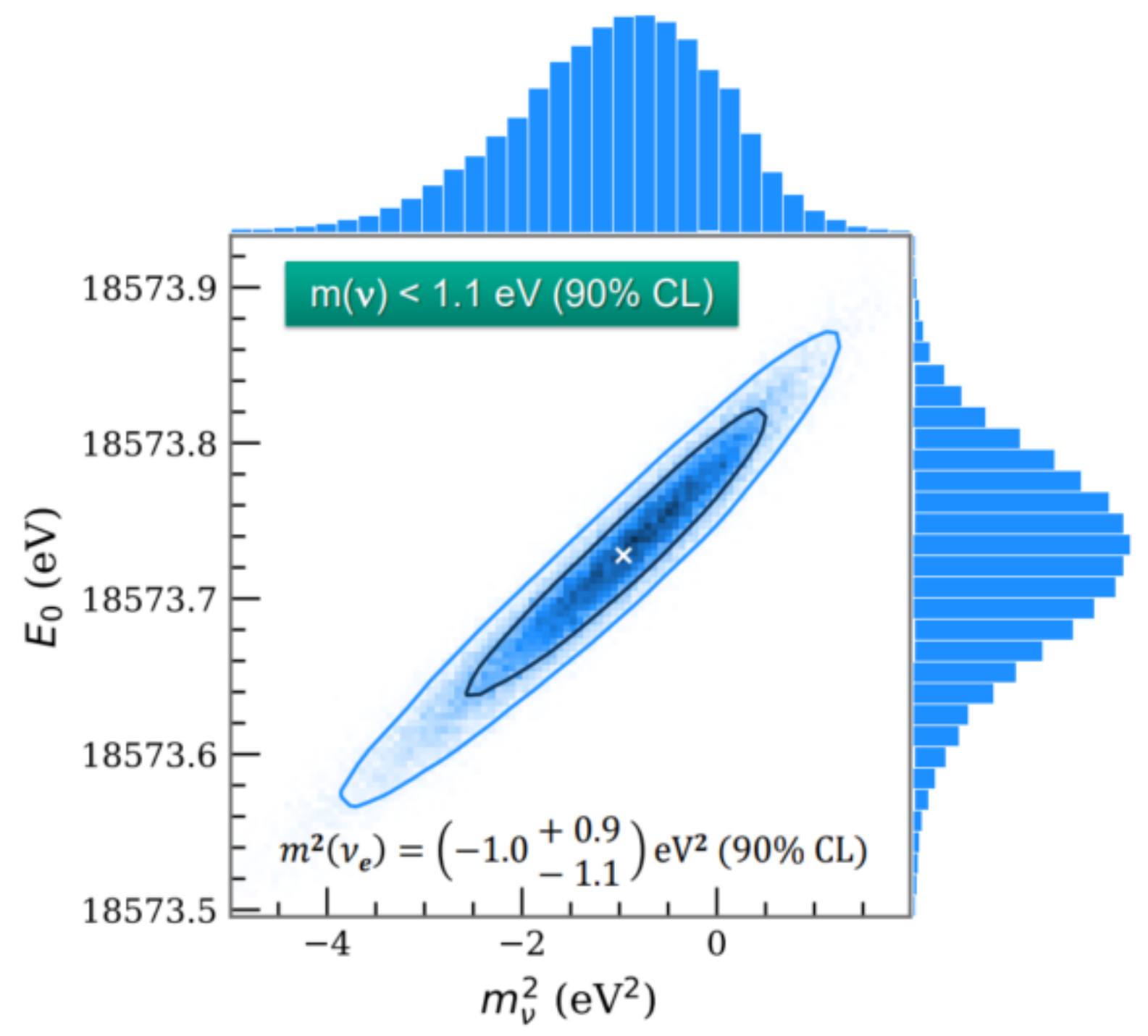

Figure 1. KATRIN: $m(\nu)<1.1 \mathrm{eV}(90 \% \mathrm{CL})$. The most relevant thing about the new result is that KATRIN seems to be working perfectly.

\section{Neutrino mass from Planck data}

Neutrino mass is also of cosmic importance. Despite their minuscule mass, so many neutrinos were born during the Big Bang that their collective gravity influenced how all the matter in the universe clumped together into stars and galaxies. By measuring the cosmic clumpiness, cosmologists can infer the neutrino's mass. Current cosmological observations already provide the tightest bounds on the sum of the neutrino masses, although they are unable to go beyond a very tight upper limit. For $k \gg k_{f s}$, neutrinos with large thermal velocities will not contribute to the clustering of structures, but at $k \ll k_{f s}$, neutrinos behave like a cold dark matter (CDM) component. Where, $k_{f s}=0.018 \Omega_{m}^{1 / 2}\left[\Sigma m_{\nu} /(1 \mathrm{eV})\right] h M p c^{-1}$ is the neutrino free-streaming scale.

Posterior distributions on the neutrino mass sum from combinations of various cosmological data sets as of early 2017, including measurements of the power spectrum of BOSS DR12 galaxies and cosmic microwave background (CMB) measurements from the Planck 2015 data release [7], see Figure 3. 


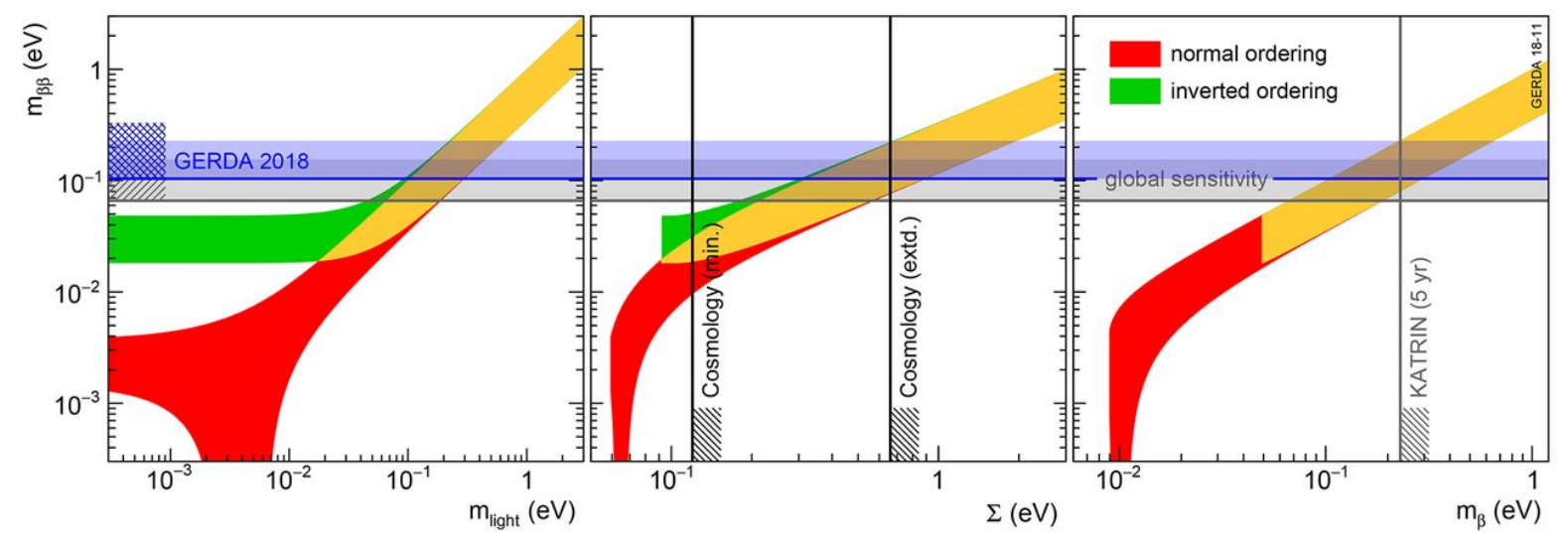

Figure 2. GERDA: Upper limit to the mass of the effective Majorana neutrino combined result is $0.07 \mathrm{eV}$ to $0.16 \mathrm{eV}$.

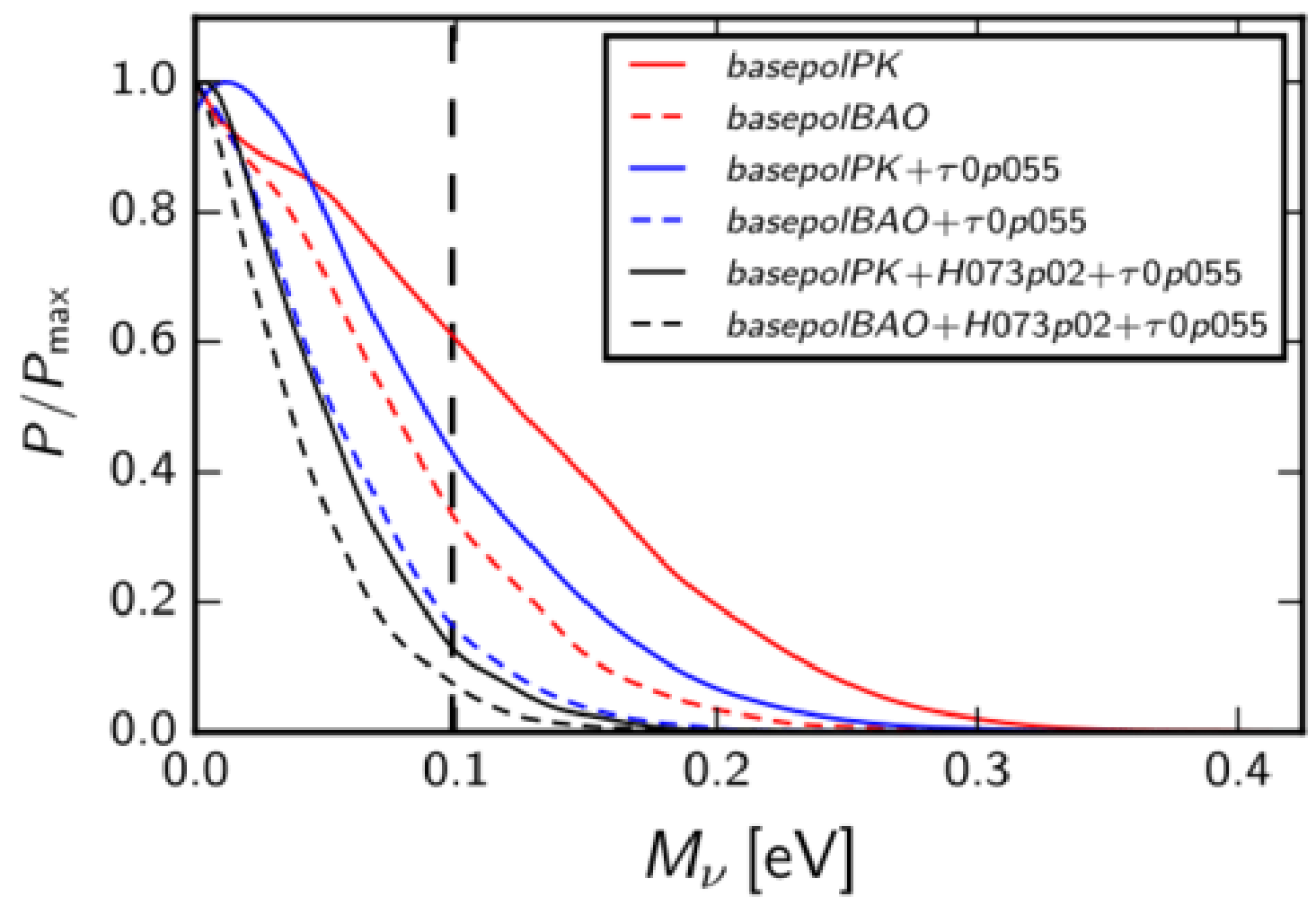

Figure 3. Old Planck 2015 data release.

From the joint analysis of the Planck cosmic microwave background (CMB) and Baryon Oscillation Spectroscopic Survey (BOSS) final data release (2019), the total neutrino mass bounds [8]:

$$
\begin{gathered}
\Sigma m_{\nu}<0.18 \mathrm{eV}(N H, \text { Planck }+F S), \\
\Sigma m_{\nu}<0.21 \mathrm{eV}(I H, \text { Planck }+F S),
\end{gathered}
$$


these values can be compared with the Planck + BAO results:

$$
\begin{aligned}
& \Sigma m_{\nu}<0.15 \mathrm{eV}(N H, \text { Planck }+B A O), \\
& \Sigma m_{\nu}<0.18 \mathrm{eV}(\text { IH, Planck }+B A O) .
\end{aligned}
$$

\section{Future cosmological probes for neutrino mass}

The cosmological data allow us to estimate the $N_{\text {eff }}$ number and the sum total of the mass $\Sigma m_{\nu}$ of ultra-relativistic particles (low-mass particles that move at almost the speed of light in a vacuum) that are in thermal equilibrium. The only particles of this type that we know of are neutrinos. For this reason, it is often said that $N_{\text {eff }}$ and $\Sigma m_{\nu}$ are cosmological observables for neutrinos from CMB Lensing. Next-generation surveys of Galaxy Clustering (the Simons Observatory [9] or CMB-S4 [10]), the effect of massive neutrinos in non-linear scales can provide the tightest constraints on the sum of neutrino masses. Also, complementary to CMB lensing and galaxy clustering the Optical Lensing can disentangle the effects of nonstandard dark energy from non-zero neutrino mass.

Future CMB clusters detection is through the thermal Sunyaev-Zel'dovich (tSZ) signal can provide useful insights on $\Sigma m_{\nu}$. Next-generation surveys will use two calibrations, internally via CMB halo lensing and externally via optical weak lensing. The systematic uncertainties will be reduced and give narrow constraints on $\Sigma m_{\nu}$, it can also reduce the physical degeneracy between $\Sigma m_{\nu}$ and dark energy parameters. Future high signal-to-noise measurements of the kinematic Sunyaev-Zel'dovich (kSZ) effect can constrain the $\Lambda$ CDM model and the sum of the neutrino masses.

There are clouds of hydrogen gas between distant quasars and the earth, that absorb ultraviolet light as the light from distant quasars travels towards us, this phenomenon, known as the Lyman- $\alpha$ forest, is a unique probe of the growth of structure on small scales. It covers a redshift range, that is inaccessible by current galaxy surveys. This will provides one of the tightest constraints on $\Sigma m_{\nu}$, through future surveys such as DESI [11].

The statistical properties of Cosmic Voids can be used to break the degeneracy between $\Sigma m_{\nu}$ and $\sigma_{8}$, the amplitude of matter fluctuations on $8 h^{-1} M p c$ scales. So, the future looks good, see Figure 4. Cosmology is likely to be the first experimental avenue to move from a tight upper limit to a clear detection of $\Sigma m_{\nu}$.

Redshift dependence sensitive towards geometrical Baryon Acoustic Oscillations (BAO) and tomographic measurements of the late-time universe will break the degeneracy between $\sigma\left(\Sigma m_{\nu}\right)$ and dark energy equation of state parameter $w$, which will increase the efficiency of neutrino mass estimations from cosmology.

For the first direct detection of the cosmic neutrino background (e.g. the PTOLEMY experiment [12]), experimental efforts are also being devoted, which is a very challenging task. Together future CMB and large scale structure (LSS) will guarantee detection of the sum of neutrino masses almost $3 \sigma$ with a sensitivity of $\sigma\left(\Sigma m_{\nu}\right) \sim 14 \mathrm{meV}$, which could possibly point out evidence for Beyond the Standard Model (BSM) physics. See [1] and references within.

\section{Individual neutrino mass eigenstates from cosmology}

It will not be possible to directly measure the individual neutrino masses from cosmology but the detection of a non-zero total neutrino mass at future survey is imminent, same as for neutrino mass hierarchy $[13,14]$.

In the future, if massless neutrino preferred by cosmological data or a smaller sum of neutrino masses than the minimum allowed by oscillations, the discrepancy will be a vital argument for new physics. A careful comparison between cosmological and laboratory results (for example LEGEND-200 [15]) should always be performed before combining them for joint analysis. 


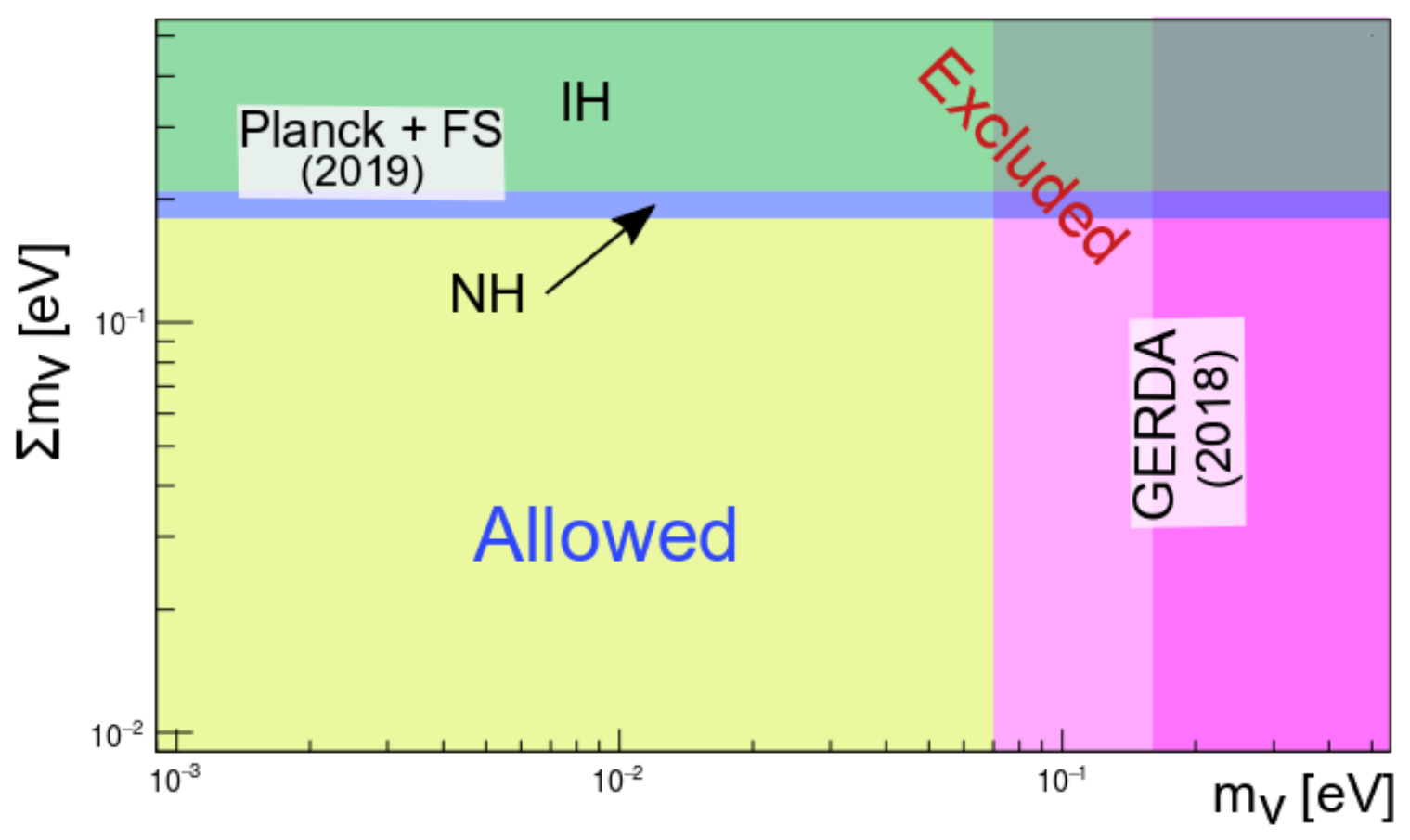

Figure 4. Comparison with laboratory experiments. Future looks good, with LSS and CMB (tSZ \& kSZ), situation will change!

[1] Dvorkin C et al. 2019 Neutrino Mass from Cosmology: Probing Physics Beyond the Standard Model arXiv:1903.03689

[2] Aker M et al. 2019 Improved Upper Limit on the Neutrino Mass from a Direct Kinematic Method by KATRIN Phys. Rev. Lett. 123221802 (arXiv:1909.06048)

[3] Aker M et al. 2020 First operation of the KATRIN experiment with tritium Eur. Phys. J. C 80264 (arXiv:1909.06069)

[4] Aghanim N et al. 2020 Planck 2018 results - VI. Cosmological parameters Astronomy ES Astrophysics 641 A6 (arXiv:1807.06209)

[5] Agostini M et al. 2019 Probing Majorana neutrinos with double- $\beta$ decay Science 3651445 (arXiv:1909.02726)

[6] Ferrario P 2017 The NEXT double beta decay experiment arXiv:1710.03022

[7] Vagnozzi S et al. 2017 Unveiling $\nu$ secrets with cosmological data: Neutrino masses and mass hierarchy Phys. Rev. D 96123503 (arXiv:1701.08172)

[8] Ivanov M M, Simonović M and Zaldarriaga M 2020 Cosmological parameters and neutrino masses from the final Planck and full-shape BOSS data Phys. Rev. D 101083504 (arXiv:1912.08208)

[9] Abitbol M H et al. 2019 The Simons Observatory: Astro2020 Decadal Project Whitepaper Bull. Am. Astron. Soc. 51147 (arXiv:1907.08284)

[10] Abazajian K et al. 2019 CMB-S4 Decadal Survey APC White Paper arXiv:1908.01062

[11] Font-Ribera A et al. 2014 DESI and other Dark Energy experiments in the era of neutrino mass measurements JCAP 05023 (arXiv:1308.4164)

[12] Betti M G et al. 2019 Neutrino physics with the PTOLEMY project: active neutrino properties and the light sterile case JCAP 07047 (arXiv:1902.05508)

[13] Archidiacono M, Hannestad S and Lesgourgues J 2020 What will it take to measure individual neutrino mass states using cosmology? JCAP 09021 (arXiv:2003.03354)

[14] Roy Choudhury S and Hannestad S 2020 Updated results on neutrino mass and mass hierarchy from cosmology with Planck 2018 likelihoods JCAP 07037 (arXiv:190\%.12598)

[15] López-Castaño J M and Guinn I 2019 Current status of LEGEND: Searching for Neutrinoless Double-Beta Decay in 76Ge: Part II arXiv:1912.03308 